\title{
Empatia, depressão, ansiedade e estresse em Profissionais de Saúde Brasileiros
}

\author{
Empathy, depression, anxiety and stress in Brazilian Health Professionals
}

\author{
Empatía, depresión, ansiedad y estrés en Profesionales de la Salud Brasileños
}

\author{
Leonardo Rodrigues Sampaio ${ }^{1}$, ORCID 0000-0003-2383-4094 \\ Letícia Coelho de Oliveira ${ }^{2}$, ORCID 0000-0002-5299-9894 \\ Michelle França Dourado Neto Pires ${ }^{3}$, ORCID 0000-0002-0770-9528 \\ ${ }^{12}$ Universidade Federal do Vale do São Francisco (UNIVASF). Brasil \\ ${ }^{3}$ Faculdade Maurício de Nassau (UNINASSAU). Brasil
}

\begin{abstract}
Resumo: O presente estudo investigou as relações entre empatia, depressão, ansiedade e estresse em profissionais de saúde brasileiros. Duzentos participantes (87\% mulheres), com idades entre 22 e 67 anos $(M=35,1 ; D P=9,7)$ responderam o Índice de Reatividade Interpessoal (Davis, 1983), o Inventário de Sintomas de Estresse (Lipp, 2000), o Inventário de Depressão de Beck e o Inventário de Ansiedade de Beck. Foram observadas correlações positivas entre depressão, ansiedade e estresse, assim como entre ansiedade, depressão, angústia pessoal e fantasia. A análise de regressão indicou que aspectos cognitivos da empatia podem prever sintomas de depressão. De forma geral, os resultados sugerem que a empatia pode estar associada à Saúde Mental dos profissionais de saúde. Discute-se a necessidade de estar atento a essa relação específica, considerando sua importância para a prática profissional desses trabalhadores, assim como para pesquisas na área de Psicologia da Saúde e outras afins.
\end{abstract}

Palavras-chave: ansiedade; depressão; estresse; empatia; profissionais de saúde

\begin{abstract}
The present study investigated the relationships among empathy, depression, anxiety, and stress among Brazilian Health Professionals (BHP). Two hundred participants (87\% women), aged 22 to 67 (M $=35.1 ; S D=9.7$ ) responded the Interpersonal Reactivity Index (Davis, 1983), the Stress Symptoms Inventory (Lipp, 2000), the Beck Depression Inventory, and the Beck Anxiety Inventory. Positive correlations among depression, anxiety and stress were found, as well as among anxiety, depression, personal distress and fantasy. Regression analysis indicated that the cognitive aspects of empathy can predict symptoms of depression. Overall, results suggest that empathy can be associated with the Mental Health of the BHP. The paper discusses the need to be attentive for this specific relationship, considering its importance for professional practices of these workers, as well as for research on the field of Psychology of Health and other related fields.
\end{abstract}

Keywords: anxiety; depression; stress; empathy; health professionals

Resumen: El presente estudio investigó las relaciones entre empatía, depresión, ansiedad y estrés en Profesionales de la Salud Brasileños (PSB). Doscientos participantes (87\% mujeres), de 22 a 67 años ( $M$ $=35.1 ; D E=9.7)$ respondieron el Índice de Reactividad Interpersonal (Davis, 1983), al Inventario de Síntomas de Estrés (Lipp, 2000), al Inventario de Depresión de Beck y el Inventario Beck de Ansiedad. Se observaron correlaciones positivas entre la depresión, la ansiedad y el estrés, así como entre la ansiedad, la depresión, la angustia personal y la fantasía. El análisis de regresión indicó que los aspectos cognitivos de la empatía podrían predecir los síntomas de la depresión. En general, los resultados sugieren que la empatía podría estar asociada con la Salud Mental de PSB. Se discute la necesidad de estar atento a esta relación específica, considerando su importancia para las prácticas profesionales de este público, como para la investigación en Psicología da Salud y otros campos relacionados.

Palabras clave: ansiedad; depresión; estrés; empatía; profesionales de la salud

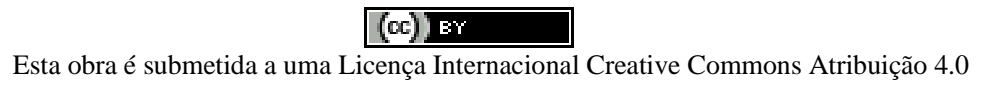


Como citar este artigo:

Sampaio, L. R., Oliveira, L. C. d, y Pires, M. F. D. N. (2020). Empatia, depressão, ansiedade e estresse em Profissionais de Saúde Brasileiros. Ciencias Psicológicas, 14(2), e-2215. doi: https://doi.org/10.22235/cp.v14i2.2215

Correspondência: Leonardo Rodrigues Sampaio, Universidade Federal do Vale do São Francisco - Univasf. Colegiado de Psicologia. Avenida José de Sá Maniçoba, s/n. Centro. Petrolina - PE, Brasil. CEP: 56304-917. Email: leorsampaio2012@gmail.com; Letícia Coelho de Oliveira, Avenida Cardoso de Sá, 1125, Edf. Saint Michelle, apt. 203, centro, Petrolina - PE, Brasil.CEP 56302-110.E-mail: leticiapnz@gmail.com; Michelle França Dourado Neto Pires, Faculdade Maurício de Nassau - Uninassau. Av. Clementino Coelho, 714. Centro, Petrolina - PE, 56308210.E-mail: chelle_fd@hotmail.com

Das dez principais causas de incapacitação em todo o mundo investigadas pela Organização Mundial da Saúde (OMS) cinco delas estão associadas aos Transtornos Mentais, destacando-se a depressão (13\%) seguida de ingestão de álcool (7\%), transtornos afetivos (3,3\%), esquizofrenia (4\%) e transtorno obsessivo-compulsivo (2,8\%). Países em desenvolvimento como o Brasil apresentam alta prevalência de problemas de saúde mental nos cuidados primários de saúde, que se refletem em altas proporções de Transtornos Mentais, especialmente ansiedade e depressão (Carlotto, 2016; Gonçalves et al., 2014; Margis, Picon, Cosner \& Silveira, 2003).

Ainda não há estudos epidemiológicos com grande abrangência que determinem a verdadeira magnitude dos Transtornos Mentais na população brasileira (Aquino, 2012). Apesar disso, sabe-se que a ansiedade e a depressão estão elencadas como as grandes síndromes psiquiátricas, ou seja, fazem parte dos maiores sofrimentos mentais que acometem as pessoas no Brasil (Dalgalarrondo, 2008).

A ansiedade é definida como um estado emocional de expectativa diante da possibilidade de algo ruim acontecer (Alves, 2012), sendo considerada transtorno quando seus níveis são excessivos ou persistem por longo período (American Psychiatric Association, 2013), a partir de quando ela pode ser classificada em dois grandes grupos: ansiedade constante/ permanente e crises de ansiedade abruptas, mais ou menos intensas (Dalgalarrondo, 2008). Diversos fatores podem desencadear a ansiedade, desde desequilíbrios químicos cerebrais, características de personalidade, vulnerabilidade genética, além de eventos traumáticos (Mangolini, Andrade \& Wang, 2019). Por sua vez, a depressão é descrita pelo DSM 5 como um transtorno afetivo que tem como elementos mais evidentes o humor triste, vazio ou irritável, acompanhado de alterações somáticas e cognitivas, que afetam significativamente a capacidade de funcionamento do indivíduo, sendo tratada nos dias atuais como problema prioritário de saúde pública, pois é a primeira causa de incapacidade ao redor do mundo (World Health Organization, 2017).

A depressão pode ser do tipo episódica ou de transtorno, sendo a segunda uma manifestação recorrente da primeira. É importante salientar que os sintomas ansiogênicos e depressivos podem ocorrer juntos, quando o transtorno passa a ser considerado misto. Nesse caso nenhuma das duas síndromes é grave o suficiente para constituir um diagnóstico fechado (CID-10, 1993).

O estresse está comumente associado aos transtornos de depressão e ansiedade, sendo geralmente identificado como uma reação disfuncional quando em excesso, que o indivíduo apresenta quando tem que lidar com uma situação desafiadora (Adriano et al., 2017; Godoy, 
Rossignoli, Delfino-Pereira, Garcia-Cairasco \& Umeoka, 2018). Essa reação é constituída por componentes físicos e psicológicos, sendo os físicos mais comuns: aumento da sudorese, fadiga, dores de cabeça, insônia, dores no corpo, palpitações, ranger de dentes, alterações intestinais, náusea, tremores, extremidades frias e resfriados constantes (Costa \& Pinto, 2017). Entre os sintomas psíquicos destacam-se a diminuição da concentração e memória, indecisão, confusão, perda do senso de humor, ansiedade, dificuldade de relaxar, nervosismo, depressão, raiva, frustração, preocupação, medo, irritabilidade, impaciência, vontade de abandonar tudo e angústia (Fabri et al., 2018; Ferreira \& Martino, 2006; Leão et al., 2017).

No que se refere especificamente ao contexto brasileiro, estudos anteriores indicam a presença de transtornos do sono, alta frequência de distúrbios relacionados à ansiedade e depressão e de níveis elevados de estresse entre médicos, enfermeiros e outros profissionais da área da saúde (Ferreira \& Martino, 2006; Batista \& Bianchi, 2006; Moura et al., 2018; Santos \& Cardoso, 2010).

Por exemplo, a pesquisa realizada por Lourenção et al. (2017) demonstrou que médicos residentes no primeiro ano de formação tendiam a apresentar sintomas de depressão e distúrbios de ansiedade, e que havia uma relação entre a ansiedade e o desejo de desistir do programa de residência. Outros estudos indicam que no caso específico dos enfermeiros, os fatores desencadeantes do estresse e da depressão podem estar associados ao local de trabalho, tais como o setor de atuação, turno, sobrecarga de serviço, mas também a fatores como sexo, idade, carga de trabalho doméstico, suporte e renda familiar, além de outras características individuais (Ferreira \& Martino, 2006; Pereira et al., 2017; Schmidt, Dantas \& Marziale, 2011). Sobre este último ponto, o estilo atribucional das pessoas é apontado como o principal fator que predispõe ao adoecimento, pois os eventos negativos da vida são interpretados como estando sob seu controle e responsabilização, bem como há uma supervalorização desses. Além disso, eventos negativos ganham um caráter de durabilidade e persistência na consciência das pessoas acometidas pela depressão, levando-as a um estado de onipotência (Alves, 2012).

Marchi, Bárbaro, Miasso e Tirapelli (2013) administraram o Inventário de Ansiedade de Beck (BAI) a uma amostra de 308 estudantes de enfermagem de uma universidade pública brasileira e observaram que $30 \%$ dos participantes apresentou grau mínimo de ansiedade, 34\% ansiedade leve, $24 \%$ moderada e $12 \%$ ansiedade considerada grave. A rotina de estudos e as novas experiências na área da clínica e o confronto com a morte são apontados pelos autores como possíveis explicações para os níveis de ansiedade moderada à grave. Em outro estudo no qual se administrou a BAI à uma amostra de 211 enfermeiros, houve uma prevalência de 31,3\% de ansiedade (Schmidt et al., 2011).

Essas evidências científicas apontam para o impacto que as demandas às quais os profissionais de saúde são submetidos diariamente - lidando com situações extremas de cuidado e atenção e tendo que conviver com o sofrimento de outrem - podem ter para sua própria saúde mental. Não somente aspectos relacionados às condições externas, pois fatores de ordem constitucional também exercem influência no surgimento e agravos de transtornos psíquicos (Fernandes, Falcone \& Sardinha, 2012).

É razoável propor hipóteses que associem competências sociais como a empatia à uma maior habilidade para manejar os próprios sentimentos negativos, desencadeados a partir da observação do sofrimento das outras pessoas. Esta suposição apoia-se em evidências que demonstram que os componentes cognitivos e afetivos da empatia são essenciais para que o sujeito seja capaz de compreender os estados emocionais das outras pessoas e possa compartilhar 
vicariamente estas experiências (Berliner \& Masterson, 2015). Ainda, os componentes comportamentais da empatia (expressões que informam sobre a compreensão do estado emocional do outro) permitem que o indivíduo atue de forma mais eficaz sobre os fatores que causam sofrimento em outrem (Falcone, 2015), o que pode ser especialmente importante para profissionais de saúde.

Corroborando estas suposições, estudos anteriores demonstram que o comprometimento no desenvolvimento da empatia tem sido relacionado à dificuldades no manejo da raiva, transtornos da personalidade e ansiedade crônica (Falcone, 2015). Além disso, há evidências que apontam para relações entre ansiedade, depressão e comportamento empático reduzido entre profissionais de saúde (Bordin et al., 2019). Por esta razão, Schreiter, Pijnenborg e Rot (2013) apontam que a pobreza de habilidades empáticas seria uma das razões pelas quais indivíduos com depressão apresentam prejuízo nas funções sociais. A partir de uma ampla revisão de literatura, esses autores constataram que os componentes cognitivos da empatia estão comprometidos em pessoas depressivas, enquanto a angústia empática (componente afetivo relacionado à experiências de sofrimento e angústia experimentadas no self) estaria elevada. $\mathrm{O}$ nível mais alto da angústia empática está associado justamente ao comportamento de afastamento e esquiva das interações sociais, presente em pessoas acometidas pela depressão.

Em concordância com esses dados, o estudo realizado por Alves (2012) com estudantes universitários identificou que maiores níveis de angústia pessoal estavam associados a maiores níveis de depressão e ansiedade, enquanto que menores níveis de tomada de perspectiva eram observados mais frequentemente em participantes com maior prevalência de sintomas depressivos, do que em indivíduos com baixa prevalência.

Partindo de outra perspectiva, Barbosa et al. (2013) demonstraram que um treinamento específico para desenvolvimento da empatia pode contribuir para diminuição da ansiedade e um aumento na empatia em estudantes de cursos da área da saúde. Por esta razão, alguns autores sugerem que a empatia pode atuar como uma espécie de fator de proteção à saúde mental, influenciando a maneira como as pessoas avaliam seu contexto de trabalho e lidam com situações de estresse (Lee, Brennan \& Daly, 2001).

Portanto, conhecer como a saúde mental pode estar relacionada à empatia se faz relevante, tendo em vista que esse conhecimento pode contribuir para o desenvolvimento de estratégias mais eficazes de promoção à saúde. No que se refere mais especificamente aos profissionais que atuam na área da saúde, essa compreensão pode direcionar a sua prática para que os serviços oferecidos sejam mais eficazes e humanizados. Além disso, compreende-se que a implantação de estratégias baseadas em evidências tem como consequência, além dos benefícios para os próprios profissionais, incrementos na qualidade dos serviços oferecidos à população atendida na rede de assistência à saúde.

Face ao exposto, no presente trabalho partiu-se da perspectiva de que a empatia é um componente importante da vida em sociedade, por permitir o compartilhar de experiências afetivas e a tomada de perspectiva das pessoas à nossa volta. Conforme já consagrado na literatura, este componente tem papel essencial na maneira como os indivíduos se relacionam uns com os outros e com o ambiente social (Eisenberg, Fabes \& Spinrad, 2006; Hoffman, 2007), promovendo maior engajamento em comportamentos prosociais (Paciello, Fida, Cerniglia, Tramontano \& Cole, 2013; Wang, Wang, Deng \& Chen, 2019). Assim, nesse estudo buscou-se avaliar a relação entre empatia, ansiedade, estresse e depressão em profissionais que atuam na rede de assistência à saúde de um 
município do interior do Nordeste brasileiro. Além disso, verificou-se em que medida as dimensões da empatia poderiam ser usadas como preditoras da ansiedade e da depressão.

\section{Método}

\section{Amostra}

Participaram da pesquisa 221 profissionais que atuam na rede pública de saúde no município de Petrolina (PE) - Brasil, há pelo menos dois anos e que se disponibilizaram a responder os instrumentos. Porém, na análise de dados foram utilizados apenas os protocolos de 200 participantes ( $87 \%$ mulheres, $n=174)$, com idades variando entre 22 e 67 anos $(M=35,1$ e $D P=9,7)$, em decorrência do não preenchimento de algum dos instrumentos ou desistência durante a coleta de dados.

Em relação à profissão de formação, $43 \%$ eram técnicos de enfermagem $(n=86), 37 \%$ eram enfermeiros $(n=75), 16 \%$ médicos $(n=32)$ e 3,5\% odontólogos $(n=7)$. Destes, $47 \%$ trabalhavam em hospitais $(n=94), 22,5 \%$ em Unidades de Atenção Básica à Saúde $(n=45), 9 \%$ no Serviço de Atendimento Móvel de Urgência - SAMU $(n=18)$ e 21,5\% em outros locais $(n=$ 43). Em relação ao regime de trabalho, a maioria $(50,5 \% ; n=101)$ declarou ser diarista, $30 \%(n$ $=60)$ plantonista e $37 \%(n=74)$ assumiram ter ambos os regimes de trabalho. Quanto à quantidade de horas trabalhadas por semana, 51,6\% $(n=103)$ afirmaram trabalhar até 40 horas por semana e o restante assumia cargas horarias que iam até 168 horas por semana.

\section{Instrumentos e materiais}

Para mensuração da empatia foi utilizado o Interpersonal Reactivity Index (IRI) de Davis (1983), que é um instrumento autoavaliativo composto por 28 itens distribuídos em duas dimensões cognitivas (Tomada de Perspectiva e Fantasia) e duas afetivas (Angústia Pessoal e Consideração Empática). A Tomada de Perspectiva corresponde à capacidade de se colocar no lugar de outras pessoas, assumindo o seu ponto de vista. A Fantasia envolve uma capacidade similar, porém aplicada a personagens fictícios de filmes, novelas e livros. A angústia pessoal se refere a um tipo de sentimento empático experienciado no self como uma experiência negativa que causa incômodo e desconforto no próprio indivíduo, quando ele se depara com o sofrimento ou infortúnio de outrem. Por fim, a Consideração Empática é conceituada como um tipo de sentimento que mobiliza o indivíduo a se engajar em ações para diminuir a condição que causa sofrimento ou infortúnio na pessoa por quem o indivíduo sente empatia. No presente estudo, foi utilizada a versão do IRI adaptada no Brasil e validada por Sampaio, Guimarães, Camino, Formiga e Menezes (2011), que contém apenas 26 itens.

Para investigar a sintomatologia do estresse nos participantes, foi utilizado o Inventário de Estresse para Adultos de Lipp - ISSL (Lipp, 2000), composto por 37 itens que correspondem a sintomas de natureza somática e 19 psicológicas, organizados em três quadros que permitem a classificação de diferentes fases do estresse. O primeiro quadro é constituído por 15 itens que se referem aos sintomas físicos ou psicológicos experimentados pelo respondente nas últimas 24 horas. O segundo é composto de dez sintomas físicos e cinco psicológicos, que podem ter sido vivenciados na última semana. O terceiro quadro, composto por 12 sintomas físicos e 11 psicológicos, refere-se a sintomas experimentados no último mês. O estresse pode ser classificado 
como presente ou ausente e nos casos em que ele está presente verifica-se a fase na qual seus sintomas estão acontecendo: alerta, resistência, quase-exaustão e exaustão.

A depressão e a ansiedade foram avaliadas por meio do Inventário de Depressão de Beck (BDI) e do Inventário de Ansiedade (BAI), respectivamente, ambos desenvolvidos por Beck e seus colaboradores. O BDI é um instrumento composto por 21 itens relacionados a sintomas presentes na depressão (comportamentais, cognitivos, afetivos e somáticos), os quais permitem classificar a doença como Mínima, Leve, Moderada ou Grave. Já a BAI é constituída por 21 itens referentes a sintomas comuns em quadros de ansiedade, nos quais se solicita que o respondente indique o quanto foi acometido por cada sintoma durante a semana que passou, numa escala de 4 pontos, variando de 0 (não) a 3 (severamente). O escore total do BDI pode variar de 0 a 63, a partir da soma dos itens. A ansiedade pode ser classificada como Mínima, Leve, Moderada ou Grave. No presente estudo empregou-se as versões do BDI e do BAI adaptadas para a população brasileira por Cunha (2001).

Além desses instrumentos, os participantes também responderam a um questionário com questões sobre seu ambiente e rotinas de trabalho, bem como dados sociodemográficos.

\section{Procedimentos de coleta de dados}

As escalas e os questionários foram encadernados em um volume único contendo 12 páginas, com os instrumentos organizados na seguinte ordem: primeiro o Termo de Consentimento Livre e Esclarecido, seguido das escalas BAI, BDI, IRI, ISSL e questionário sociodemográfico. Os participantes foram abordados pelos pesquisadores em seus locais de trabalho, receberam explicações sobre o estudo e após concordarem em participar indicavam o melhor horário para coleta de dados. Os instrumentos foram preenchidos de forma individual, nos locais e horários de melhor conveniência para os respondentes.

Todos os procedimentos adotados no presente estudo seguiram os preceitos éticos exigidos na resolução 466/2012 do Conselho Nacional de Saúde e da Resolução 016/2000 do Conselho Federal de Psicologia, tendo o presente projeto sido aprovado por um Comitê de Ética em Pesquisas com seres humanos (protocolo nº 0014/131113 CEDEP/UNIVASF).

\section{Análise dos dados}

A análise dos dados envolveu o uso de estatísticas descritivas (percentuais, médias e desvios-padrões) e de testes inferenciais como a Análise de Variância e o Teste de Correlação de Pearson, para avaliar as relações entre as principais variáveis do estudo. Adicionalmente, foram realizadas Análises de Regressão Múltipla, para testar o poder preditivo da empatia sobre a Ansiedade e a Depressão. Para todos os testes, adotou-se um valor máximo de 5\% no nível de significância, para rejeição da hipótese nula. O programa SPSS (versão 20) foi empregado para realização das análises estatísticas.

\section{Resultados}

No que se refere aos indicadores de saúde mental, $23 \%$ dos participantes foram classificados no nível leve de ansiedade, $8 \%$ no moderado e $3 \%$ no grave. Os demais participantes apresentaram pontuação correspondente ao grau mínimo de ansiedade. Quanto à depressão, 23\% 
dos participantes foram classificados no nível leve e 7,5\% no moderado. Não se observou nenhum caso grave de depressão.

Quanto às respostas para o Estresse, cerca de $42 \%$ da amostra apresentou estresse em alguma fase (alerta, resistência, quase-exaustão ou exaustão), estando a maioria na fase de Resistência (32\%). Vários participantes relataram sintomas acima dos limites em mais de uma fase (Tabela 1). Neste caso, Lipp (2000) indica que o estresse está em processo de agravamento e que deve, em breve, caso não haja nenhuma intervenção, se agravar para a fase seguinte. Um teste do Qui-quadrado indicou que os indicadores de saúde mental não variaram significativamente entre as categorias profissionais $(p>, 005)$.

Tabela 1

Prevalência De Estresse Na Amostra

\begin{tabular}{lc}
\hline CONDIÇÃO & $N(\%)$ \\
\hline Não presença de estresse & $116(58)$ \\
Resistência & $64(32)$ \\
Resistência / Exaustão & $8(4)$ \\
Alerta / Resistencia / Quase-exaustão & $6(3)$ \\
Alerta / Resistencia & $4(2)$ \\
Alerta & $2(1)$ \\
Quase-exaustão / Exaustão & $1(0,5)$ \\
Exaustão & $1(0,5)$ \\
\hline
\end{tabular}

O Teste de Pearson indicou a existência de correlações positivas e estatisticamente significativas entre ansiedade e depressão e entre depressão e estresse, conforme demonstrado na Tabela 2. 
Tabela 2

Correlações Entre Os Indicadores De Saúde Mental E As Dimensões Da Empatia

\begin{tabular}{lcccccccc}
\hline & $(1)$ & $(2)$ & $(3)$ & $(4)$ & $(5)$ & $(6)$ & $(7)$ & (8) \\
\hline Ansiedade (1) & - & & & & & & \\
Depressão (2) &, $51^{*}$ & - & & & & & \\
Estresse (3) &, $29^{* *}$ &, $51^{* *}$ & - & & & & \\
Empatia global (4) &, $18^{*}$ &, $17^{*}$ & n.s. & - & & & & \\
Tomada de Perspectiva (5) & n.s. & n.s. & n.s. &, $66^{* *}$ & - & & \\
Consideração Empática (6) & n.s. & n.s. & n.s. &, $82^{* *}$ &, $62^{* *}$ & - & \\
Angústia Pessoal (7) &, $29^{* *}$ &, $24^{* *}$ & n.s. &, $76^{* *}$ &, $3 *$ &, $47^{* *}$ & - & \\
Fantasia (8) &, $32^{* *}$ &, $26 * *$ & n.s. &, $79^{* *}$ &, $3 * *$ &, $49^{* *}$ &, $97^{* *}$ & - \\
\hline
\end{tabular}

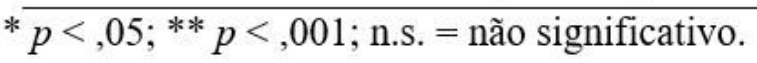

No que se refere especificamente à Empatia, a Consideração Empática foi a dimensão com maior pontuação média $(M=4,10 ; D P=0,65)$, seguida de Tomada de Perspectiva $(M=4,06 ; D P$ $=0,62)$, Angustia Pessoal $(M=2,80 ; D P=0,83)$ e Fantasia $(M=2,73 ; D P=0,80)$. Para verificar se havia diferenças nos indicadores de Empatia em função do sexo e da categoria profissional dos participantes procedeu-se com uma ANOVA, seguida de um post-hoc test de Tukey. Os resultados desses testes apontam para uma influência significativa da profissão na subdimensão de Tomada de Perspectiva $\left[\mathrm{F}(3,199)=3,307 ; p=, 021 ; \eta^{2}=0.04\right)$, com os médicos pontuando menos $(M=$ $3,76 ; D P=0,67)$ do que os técnicos de enfermagem $(M=4,15 ; D P=0,65)$.

Conforme esperado, todas as dimensões da Empatia se correlacionaram positivamente entre si e com a Empatia Global (Tabela 2). Além disso, foi verificada a existência de correlações positivas entre Ansiedade e Depressão com a Empatia Global e de correlações positivas entre Angustia Pessoal e Fantasia com Ansiedade e Depressão.

Para testar em que medida as dimensões de Empatia poderiam ser usadas como preditoras da Ansiedade e da Depressão, foram realizadas análises de regressão múltipla (método stepwise), tendo como variáveis dependentes Ansiedade e Depressão, e a Angustia Pessoal, Tomada de Perspectiva, Consideração Empática e Fantasia como variáveis independentes. Os resultados apresentados nas Tabelas 3 e 4 indicam que a Ansiedade foi predita positivamente por Fantasia e inversamente por Tomada de Perspectiva, com ambas explicando cerca de $11 \%$ da variância total observada. Além disso, a Depressão foi predita de forma positiva por Fantasia e Consideração Empática, e inversamente por Tomada de Perspectiva. 
Tabela 3

Regressão Linear Múltipla Para A Predição Da Ansiedade

\begin{tabular}{lcccccc}
\hline Variáveis & $\mathrm{R}$ & $\mathrm{R}^{2}$ & $\mathrm{~F}$ & $\mathrm{Sig}(\mathrm{F})$ & Beta & $\mathrm{t}$ \\
\hline Fantasia &, 35 &, 11 & 13,83 &, &, 32 & 4,76 \\
Tomada de Perspectiva & & & &, &,- 15 & $-2,14$ \\
\hline
\end{tabular}

Tabela 4

Regressão Linear Múltipla Para A Predição Da Depressão

\begin{tabular}{lcccccc}
\hline Variáveis & $\mathrm{R}$ & $\mathrm{R}^{2}$ & $\mathrm{~F}$ & $\mathrm{Sig}(\mathrm{F})$ & $\mathrm{Beta}$ & $\mathrm{t}$ \\
\hline Fantasia &, 35 &, 11 & 9,64 &, &, 26 & 3,86 \\
Tomada de Perspectiva & & & &, &,- 21 & $-2,97$ \\
Consideração Empática & & & &, &, 19 & $-2,4$ \\
\hline
\end{tabular}

\section{Discussão}

Os resultados do presente estudo corroboram pesquisas anteriores (Aquino, 2012; Fabri et al., 2018; Moura et al., 2018; Santos \& Cardoso, 2010) que destacam a prevalência de agravos à saúde mental de profissionais brasileiros que atuam no campo da saúde. A prevalência de ansiedade e depressão pode ser considerada baixa (11\% e 15\%, respectivamente), em comparação com outros estudos nacionais (Schmidt et al., 2011). Porém, se destaca o fato de que quase metade da amostra (43\%) apresentou estresse em alguma fase, sendo a maior prevalência na fase de Resistencia.

A correlação positiva entre depressão e ansiedade também corrobora a literatura, que aponta uma relação clínica entre processos depressivos e ansiogênicos na população em geral (American Psychiatric Association, 2013; Aquino, 2012; CID 10, 1993; Dalgalarrondo, 2008), e também em amostras de profissionais e estudantes da área da saúde (Ferreira \& Martino, 2006; Forteney et al., 2013; Marchi et al., 2013; Mascarenhas et al., 2012; Murofuse, Abranches \& Napoleão, 2005; Ribeiro et al., 2020; Schmidt et al., 2011). Essa relação pode ser justificada pelo fato desses quadros psíquicos serem semelhantes, pois ambos se formam a partir de esquemas patológicos, carregados de sentimentos negativos em relação ao self, ao seu futuro e ao mundo. Desta forma, o indivíduo acaba construindo e reforçando representações depreciativas sobre si mesmo e sobre as pessoas à sua volta, o que torna sua leitura da realidade enviesada por conteúdos negativos (Alves, 2012; Knapp \& Beck, 2008).

Por outro lado, era esperada uma relação entre ansiedade, depressão e estresse, pois a literatura aponta para similaridades na etiologia desses transtornos (Murofuse et al., 2005), o que não foi verificado no presente estudo. É possível que esse resultado tenha ocorrido pelo fato de que no estresse os sintomas só se tornam comprometedores das rotinas habituais das pessoas em fases mais avançadas (Lipp, 2000; Mascarenhas et al., 2012). A este respeito é importante lembrar que uma parte expressiva da amostra apresentou sinais de estresse, porém em baixa magnitude, o 
que já indicaria a presença deste quadro entre os que padecem de depressão e ansiedade, mas em intensidade ainda não passível de alcançar significância estatística, quando da sua associação com as demais variáveis investigadas.

Esses resultados reforçam o argumento de que se faz necessário atentar para as demandas laborais as quais os profissionais de saúde são submetidos, desde a sua formação acadêmica, até quando estes se inserem na rede de assistência à saúde. Mais ainda quando se pensa no papel social atribuído a estes profissionais, frente à precariedade do sistema de saúde brasileiro, o que os obriga a ter um estilo de vida no qual o cuidado com sua própria saúde fica em segundo plano.

O estilo de vida é considerado um fator importante no desenvolvimento de transtornos mentais, incluindo depressão, estresse e ansiedade, agravando-se pelo fato de que diante da situação de impotência ou fracasso, os eventos negativos tendem a ser interpretados com mais importância do que os positivos. Isso leva os indivíduos a se culparem constantemente pelo que fizeram ou deixaram de fazer (Alves, 2012; Araújo, Aquino, Menezes, Santos \& Aguiar, 2003; Murofuse et al., 2005).

No que diz respeito especificamente às dimensões da Empatia, a Consideração Empática foi a que apresentou maior pontuação, seguida pela Tomada de Perspectiva. Ou seja, de modo geral os participantes pontuaram mais em componentes empáticos diretamente associados ao cuidado com o outro (Hoffman, 2007), o que pode ser de especial importância para o contexto de trabalho desses profissionais, que são demandados a lidar diariamente com o sofrimento e fragilidade daqueles que procuram seus serviços.

Além disso, foi observada uma associação significativa da dimensão Tomada de Perspectiva com a categoria profissional dos participantes, de forma que os médicos pontuaram menos que os técnicos em enfermagem. É possível que esta diferença seja reflexo do contato mais próximo e continuado que os enfermeiros desenvolvem em relação aos seus pacientes, demandando maior sensibilidade às suas condições clínicas, para que possam assisti-los de forma mais humanizada. Por outro lado, a relação entre o médico e o paciente, segundo Barros, Falcone e Pinho (2011), desde os primórdios da prática de saúde parece estar constituída em um modelo hierárquico, o que levaria a uma menor sensibilidade do profissional em relação ao paciente. Além disso, as enormes fragilidades da rede de assistência à saúde pública no Brasil, frente às demandas da população, obrigariam estes profissionais a realizar diversos atendimentos rápidos e superficiais em um mesmo turno, os quais desestimulariam a formação de vínculos mais próximos com os seus assistidos.

A este respeito, há questionamentos na forma de cuidado e tratamento dos médicos, com críticas que afirmam que os médicos tendem a ser mais "frios" e "distantes" (Benevides-Pereira \& Gonçalves, 2009; Nogueira-Martins, 2003). Porém, Aquino (2012) ressalta que esse comportamento pode estar associado à carga emocional imposta quando esses profissionais se deparam repetidamente com o sofrimento de outrem, o que os levaria a assumir uma postura mais distante e retraída em relação aos seus pacientes, como uma forma de defesa psíquica. Não se pode deixar de considerar também a sobrecarga de trabalho a que estão submetidos os médicos que atuam no Sistema Único de Saúde brasileiro - SUS (Santos, Alves, Souza, Queiroz \& Castanho, 2012), o que contribuiria ainda mais para essa despersonalização das pessoas atendidas por esses profissionais.

Confirmando a hipótese principal da pesquisa, evidenciou-se a existência de relações entre ansiedade, depressão, estresse e empatia, com os componentes dessa última variável apresentando 
capacidade preditiva sobre o surgimento de sinais da ansiedade e da depressão. Pesquisas anteriores já haviam demonstrado que quanto maiores os níveis de estresse, depressão e ansiedade, menores os índices de empatia (Aquino, 2012; Benevides-Pereira \& Gonçalves, 2009; Bordin et al., 2019; Pacheco, 2013) e que menores escores em medidas de empatia estariam associados a baixo desempenho em habilidades sociais e no engajamento em comportamentos prossociais (Pavarino, Del Prette \& Del Prette, 2005; Wang et al., 2019).

As relações entre ansiedade, depressão e estresse com angústia pessoal podem ser explicadas pelo fato de que nesta dimensão empática os fatores preponderantes são os sentimentos negativos de incômodo, perturbação e desconforto experienciados no self, quando o indivíduo se depara com o sofrimento do outro. Portanto, sujeitos com angústia pessoal mais intensa seriam mais propensos a sofrer mais com a instalação de quadros depressivos e ansiogênicos, visto que as representações mentais relacionadas a si mesmo e que envolvem conteúdos negativos seriam reforçadas. Assim, diante de uma situação na qual outrem esteja precisando de ajuda, sujeitos com sintomas de estresse, ansiedade e depressão, tenderiam a focar mais no seu sofrimento, o que impediria a mobilização do comportamento de ajuda ao outro.

A este respeito, Hoffman (2007) chama atenção para o fato de que diante de situações nas quais a angústia empática é elevada a ponto de se tornar insuportável, o indivíduo perde a capacidade de enxergar as necessidades do outro e torna-se mais egoísta, tendo dificuldade para diferenciar e regular suas próprias emoções. Assim, essa sobrecarga empática reforçaria os sintomas ansiosos e depressivos, pois, conforme indica o estudo de Batson, Fultz e Schoenrad (1987) a intensidade elevada do sentimento de angústia empática geralmente está associada a atitudes mais egoístas e a comportamentos de fuga e esquiva, para que se possa cessar o incômodo experienciado no self.

A correlação observada entre Fantasia, Depressão e Ansiedade está de acordo com o que foi encontrado no estudo desenvolvido por Alves (2012), no qual também se verificou que indivíduos com alto índice de Fantasia também apresentavam altos níveis de ansiedade. Essa relação pode ser justificada porque a Fantasia envolve a capacidade imaginativa do sujeito em relação a personagens fictícios, e no caso da depressão e ansiedade o indivíduo cria esquemas negativos imaginativos que não estão realmente ligados à realidade, mas seriam representações "fictícias" de algo que existe somente em sua própria mente. Dessa maneira, a imagem que o sujeito constitui sobre si é influenciada por representações mentais negativas que ele constrói a seu próprio respeito.

Além disso, pessoas que possuem alto nível de ansiedade tentam prever situações que ainda não ocorreram (Heimberg \& Magee, 2016), o que levaria a um estado de angústia e sofrimento pelo fato de apenas imaginar o que pode acontecer. Ou seja, o estado ansiogênico faria com que as pessoas estivessem mais propensas a sofrer com situações projetadas em seu imaginário.

Um dado importante de ser observado é o fato de a Tomada de Perspectiva ter predito inversamente a ansiedade e a depressão, visto que esta dimensão empática precisa que o indivíduo assuma o ponto de vista do outro, buscando compreender seus estados internos e sua realidade social. Dessa forma, pode-se supor que a Tomada de Perspectiva mais desenvolvida poderia auxiliar o indivíduo a regular a intensidade emocional das experiências empáticas. É possível também que a Tomada de Perspectiva esteja comprometida em sujeitos com transtornos de ansiedade e depressão, já que eles tendem a manter o foco da atenção no self, o que dificultaria a compreensão sobre o sofrimento do outro (Alves, 2012). 
Em relação ao modelo de predição envolvendo a Consideração Empática, levanta-se a hipótese de que a motivação prossocial decorrente desse sentimento empático tem um custo para o sujeito, pois quanto mais intensa é sua manifestação, maior é a probabilidade do indivíduo não conseguir colocar essa motivação em prática (considerando as circunstâncias a qual são submetidos os profissionais de saúde), o que geraria frustação e aumentaria a chance de depressão. Essa hipótese reforçaria a tese que trata de a necessidade dos profissionais de saúde bloquearem a compaixão para com o outro, para que eles mesmos não sejam assolados com a frustração produzida diante do sofrimento da população e da impossibilidade de resolver problemas estruturais inerentes à sua profissão e ao cenário brasileiro.

\section{Considerações finais}

De modo geral, algumas limitações no estudo podem ser apontadas, como por exemplo o fato de ter sido constituída uma amostra de conveniência e apesar da quantidade de participantes ter possibilitado o uso de estatísticas inferenciais, não houve um balanço entre o número de participantes do sexo feminino e masculino, como também não houve um equilíbrio entre o número de participantes de cada categorial profissional. Dessa forma, seria interessante que em estudos futuros o número de participantes nos diversos estratos pudesse ser elevado, para que análises comparativas possam ser aprofundadas.

A partir dos resultados encontrados, recomenda-se ainda que investigações futuras procurem compreender o papel que outros fatores constitucionais, tais como a resiliência e os traços de personalidade, tem para o enfrentamento do estresse ocupacional em profissionais da saúde, bem como sua associação com a empatia. A investigação desses aspectos possibilitaria responder questionamentos ainda não explorados empiricamente, concernentes não apenas ao campo da Psicologia da Saúde, mas também a outros como o do Desenvolvimento Humano e da Psicologia Social. Além disso, novos estudos podem fornecer mais subsídios que permitam direcionar as estratégias de intervenção voltadas para promoção de saúde nesses profissionais, de forma a atuar sobre variáveis cujo impacto sobre a saúde mental seja cientificamente demonstrado.

De forma geral, considera-se que os dados obtidos a partir do presente estudo podem ser utilizados na elaboração de estratégias que estimulem o desenvolvimento de habilidades empáticas mais maduras, para que diante do sofrimento do outro, o profissional seja capaz de se descentrar cognitivamente do seu próprio desconforto, e assim emitir comportamentos de ajuda, sem prejuízos emocionais para si mesmo. A implantação de estratégias com objetivo dessa natureza, desde o período de formação acadêmica dos profissionais da saúde, traria benefícios não apenas para sua saúde mental no futuro, mas também para a qualidade dos serviços prestados por eles à população.

\section{Referências}

Adriano, M. S. P. F., Almeida, M. R., Ramalho, P. P. L., Da Costa, I. P., Do Nascimento, A. R. S., \& Moraes, J. C. O. (2017). Estresse Ocupacional em Profissionais da Saúde que Atuam no Serviço de Atendimento Móvel de Urgência de Cajazeiras - PB. Revista Brasileira de Ciências da Saúde, 21, 29-34. doi: 10.4034/RBCS.2017.21.01.04. 
Alves, S. A. (2012). A relação entre capacidades empáticas, depressão e ansiedade em jovens. (Dissertação de Mestrado). Centro de Ciências Humanas, Letras e Artes, Universidade Federal da Paraíba, João Pessoa. Brasil.

American Psychiatric Association (2013). Diagnostic and Statistical Manual of Mental disorders - DSM-5. 5th.ed. Washington: American Psychiatric Association.

Aquino, M. T. (2012). Prevalência de transtornos mentais entre estudantes de medicina da Universidade Federal de Minas Gerais. (Dissertação de mestrado). Ciências da Saúde da Faculdade de Medicina da Universidade Federal Minas Gerais. Belo Horizonte. Brasil.

Araújo, T. M., Aquino, E., Menezes, G., Santos, C. O., \& Aguiar, L. (2003). Work psychosocial aspects and psychological distress among nurses. Revista de Saúde Pública, 37, 424-433. doi: 10.1590/S0034-89102003000400006

Barbosa, P.; Raymond, G.; Zlotnick, C.; Wilk, J.; Toomey, R.; \& Mitchell, J. (2013). MindfulnessBased Stress Reduction Training is Associated with Greater Empathy and Reduced Anxiety for Graduate Healthcare Students. Education for Health, 26(1), 9-14, doi: 0.4103/13576283.112794.

Barros, P. S.; Falcone, E. M. O.; \& Pinho, V. D. (2011). Avaliação da empatia médica na percepção de médicos e pacientes em contextos público e privado de saúde. Arquivos de Ciência Saúde, $18,36-43$.

Batista, K.M., \& Bianchi, E. R. F. (2006). Estresse do enfermeiro em unidade de emergência. Revista Latino-Americana de Enfermagem, 14(4), 534-539. doi: 10.1590/S010411692006000400010

Batson, C.D., Fultz, J., \& Schoenrade, P.A. (1987). Distress and empathy: two qualitatively distinct vicarious emotions with different motivational consequences. Journal of Personality, 55, 21-75. doi: 10.1111/j.1467-6494.1987.tb00426.x

Benevides-Pereira, A. M., \& Gonçalves, M. B. (2009). Transtornos emocionais e a formação em Medicina: um estudo longitudinal. Revista Brasileira de Educação Médica, 33, 10-23. doi: 10.1590/S0100-55022009000100003

Berliner, R., \& Masterson, T. L. (2015) Review of Research: Promoting Empathy Development in the Early Childhood and Elementary Classroom, Childhood Education, 91, 57-64. doi: 10.1080/00094056.2015.1001675.

Bordin, D., Vascoski, V. C., Pereira, A. R. G., Santos, C. B., Zanesco, C., \& Fadel, C. B. (2019). Relação entre empatia e qualidade de vida: um estudo com profissionais da atenção primária à saúde. Revista Mineira de Enfermagem, 23, e-1253. doi: 10.5935/1415-2762.20190101

Carlotto, M. S. (2016). Transtornos Mentais Comuns em trabalhadores de Unidades Básicas de Saúde: Prevalência e fatores associados, Psicologia Argumento, 34(85), 133-146. doi: 10.7213/psicol.argum.34.085.AO04.

CID-10 (1993). Classificação de Transtornos Mentais e de Comportamento da CID-10: Descrições clínicas e diretrizes diagnósticas. Porto Alegre: Artmed.

Costa, B.R.C., \& Pinto, I.C.J.F. (2017). Stress, Burnout and Coping in Health Professionals: A Literature Review. Journal of Psychology and Brain Studies, 1, 1-8.

Cunha, J. A. (2001). Manual da versão em português das escalas Beck. São Paulo: Casa do Psicólogo.

Dalgalarrondo, P. (2008). A afetividade e suas alterações. Psicopatologia e semiologia dos transtornos mentais. ( $2^{\mathrm{a}}$. ed.). Porto Alegre: Artmed.

Davis, M. H. (1983). Measuring individual differences in empathy: Evidence for a multidimensional approach. Journal of Personality and Social Psychology, 44, 113-136. doi: 10.1037/0022-3514.44.1.113

Eisenberg, N., Fabes, R. A., \& Spinrad, T. L. (2006). Prosocial development. Em N. Eisenberg (Vol. Ed.), W. Damon \& R. M. Lerner (Series Eds.), Handbook of child psychology: Social, emotional, and personality development (Vol. 3, pp. 646-718). New York: Wiley. 
Fabri, J. M. G., Noronha, I. R., Oliveira., E. B., Kestenberg, C. C. F., Harbache, L. M. A., \& Noronha, I. R. (2018). Estresse ocupacional em enfermeiros da pediatria: Manifestações físicas e psicológicas. Revista Baiana de Enfermagem, 32, 1-10. doi: 10.18471/rbe.v32.25070.

Falcone, E. M. O. (2015). O papel da empatia na saúde e nas relações sociais. V Seminário Internacional de Habilidades Sociais - Habilidades sociais e relações interpessoais: interfaces conceituais, metodológicas e aplicadas (Livro de programa). Universidade do Estado do Rio de Janeiro, Rio de Janeiro, Brasil.

Fernandes, C. S., Falcone, E. M. O., \& Sardinha, A. (2012). Deficiências em habilidades sociais na depressão: estudo comparativo. Psicologia: teoria e prática, 14, 183-196.

Ferreira, L. R. C., \& De Martino, M. M. F. (2006). O estresse do enfermeiro: análise das publicações sobre o tema. Revista de Ciências Médicas, 15, 241-248.

Forteney, L. Luchterhand, C. Zakletskaia, L. Zgierska, A. Rakel, D. (2013). Abbreviated mindfulness intervention for job satisfaction, quality of life, and compassion in primary care clinicians: a pilot study. The Annals of Family Medicine, 11, 412-420. doi: 10.1370/afm.1511

Godoy, L. D., Rossignoli, M. T., Delfino-Pereira, P. Garcia-Cairasco, N. \& Umeoka, E. H. L. (2018). A Comprehensive Overview on Stress Neurobiology: Basic Concepts and Clinical Implications (2018). Frontiers in Behavioral Neuroscience, 12, 1-23. doi: 10.3389/fnbeh.2018.00127

Gonçalves, D. A., Mari, J. J., Bower, P., Gask, L., Dowrick, C., Tófoli, L. F., Campos, M., Portugal, F. B., Ballester, D., \& Fortes. S. (2014) Brazilian multicentre study of common mental disorders in primary care: rates and related social and demographic factors, Cadernos de Saúde Pública, 30(3), 623-632. doi: 10.1590/0102-311X00158412.

Heimberg, R. G., \& Magee, L. (2016). Transtorno de ansiedade social. Em D. H. Barlow, (Org.), Manual clínico dos transtornos psicológicos: tratamento passo a passo (pp. 114-154). Porto Alegre: Artmed.

Hoffman, M. L. (2007). Empathy and moral development: Implications for caring and justice. Cambridge University Press.

Knapp, P., \& Beck, A. T. (2008). Fundamentos, modelos conceituais, aplicações e pesquisa da terapia cognitiva. Revista Brasileira de Psiquiatria, 30(2), 54-64. doi: 10.1590/S151644462008000600002

Leão, E.R., Dal Fabbro, D.R., Oliveira, R.B., Santos, I.R., Victor, E.S., Aquarone, R.L., Andrade, C.B., Ribeiro, V.F., Oliveira, R.C., Friedlander, R., \& Ferreira, D.S. (2017) Stress, self-esteem and well-beingamong female health professionals: A randomized clinical trial on the impact of a self-care intervention mediated by the senses. PLoS ONE, 12, e0172455. doi:10.1371/journal.pone.0172455

Lee, H. S., Brennan, P. F., \& Daly, B. J. (2001). Relationship of empathy to appraisal, depression, life satisfaction, and physical health in informal caregivers of older adults. Research in nursing \& health, 24(1), 44-56. doi: 10.1002/1098-240X(200102)24:13.0.CO;2-S

Lipp, M. E. N. (2000). Manual de inventário de estresse para adultos de Lipp (I.S.S.L.). São Paulo: Casa do Psicólogo.

Lourenção, L. G., Teixeira, P. R., Gazetta, C. E., Pinto, M. H., Gonsalez, E. G., \& Rotta, D. S. (2017). Níveis de Ansiedade e Depressão entre Residentes de Pediatria. Revista Brasileira de Educação Médica, 41, 557-563. doi: 10.1590/1981-52712015v41n4rb20160092

Mangolini, V. I., Andrade, L. H., \& Wang, Y. (2019). Epidemiologia dos transtornos de ansiedade em regiões do Brasil: uma revisão de literatura. Revista de Medicina (São Paulo), 98, 415422. doi: 10.11606/issn.1679-9836.v98i6p415-422.

Marchi, K. C., Bárbaro, A. M., Miasso, A. I., \& Tirapelli, C. R. (2013). Ansiedade e consumo de ansiolíticos entre estudantes de enfermagem de uma universidade pública. Revista Eletrônica de Enfermagem, 15(3), 731-739. doi: 10.5216/ree.v15i3.18924 
Margis, R., Picon, R., Cosner, A. F., \& Silveira, R. O. (2003). Relação entre estressores, estresse e ansiedade. Revista de Psiquiatria do Rio Grande do Sul, 25(1), 65-74. doi: 10.1590/S010181082003000400008

Mascarenhas, S. A. N., Roazzi, A., Leon, G. F., \& Ribeiro, J. L. P. (2012). Necessidade da gestão do estresse, ansiedade e depressão em estudantes universitários brasileiros. Actas do $9^{\circ}$ Congresso Nacional de Psicologia da Saúde.

Moura, A., Lunardi, R., Volpato, R., Nascimento, V., Bassos, T., \& Lemes, A. (2018). Fatores associados à ansiedade entre profissionais da atenção básica. Revista Portuguesa de Enfermagem de Saúde Mental, 19, 17-26. doi:10.19131/rpesm.0198.

Murofuse, N. T., Abranches, S. S., \& Napoleão, A. (2005). Reflexões sobre estresse e Burnout e a relação com a enfermagem. Revista Latino-Americana de Enfermagem, 13(2), 255-261. doi: 10.1590/S0104-11692005000200019.

Nogueira-Martins, L. A. (2003). Saúde mental dos profissionais de saúde. Revista Brasileira de Medicina do Trabalho, 1(1), 56-68.

Pacheco, A. C. C. L. (2013). Ansiedade, Depressão, Stresse, Estratégia De Coping e Suporte Social em Enfermeiro que trabalham por Turnos e em Horário Fixo Diurno (Dissertação de Mestrado). Psicologia Clínica e da Saúde, Universidade Lusófona de Humanidades e Tecnologias. Lisboa, Portugal.

Paciello, M., Fida, R., Cerniglia, L., Tramontano, C., \& Cole, E. (2013). High cost helping scenario: the role of empathy, prosocial reasoning and moral disengagement on helping behavior. Personality and Individual Differences, 55, 3-7. doi: 10.1016/j.paid.2012.11.004

Pavarino, M. G.; Del Prette, A.; Del Prette, Z. A. P. (2005). O desenvolvimento da empatia como prevenção da agressividade na infância. Psico, 36(2), 127-134.

Pereira, I. F., Faria, L. C., Vianna, R. S. M., Corrêa, P. D. S., Freitas, D. A., \& Soares, W. D. (2017). Depressão e uso de medicamentos em profissionais de enfermagem. Arquivos de Ciências da Saúde, 24, 70-74. doi:10.17696/2318-3691.24.1.2017.544

Ribeiro, C. F., Lemos., C. M. C., Alt, N. N., Marins, R. L. T., Corbiceiro, W. C. H., \& Nascimento, M. I. (2020). Prevalência de Fatores Associados à Depressão e Ansiedade em Estudantes de Medicina Brasileiros. Revista Brasileira de Educação Médica, 44, e021. doi: 10.1590/19815271v44.1-20190102.ING.

Sampaio, L. R.; Guimarães, P. R. B.; Camino, C.P.S; Formiga, N. S., \& Menezes. (2011). Estudos sobre a dimensionalidade da empatia: tradução e adaptação do Interpersonal Reactivity Index (IRI). Psico, 42, 67-76.

Santos, N. A.; Alves, C. S.; Souza, P. C.; \& Castanho, W. Q. S. (2012). O médico e as condições de trabalho: o caso de um pronto-socorro do interior do estado de Mato Grosso. Revista de Administração em Saúde, 54(14), 80-88.

Santos, A. F. O., \& Cardoso, C. L. (2010). Profissionais de saúde mental: estresse, enfrentamento e qualidade de vida. Psicologia: Teoria e Pesquisa, 26(3), 543-548. doi: 10.1590/S010237722010000300017

Schmidt, D. R. C., Dantas, R. A. S., \& Marziale, M. H. P. (2011). Ansiedade e depressão entre profissionais de enfermagem que atuam em blocos cirúrgicos. Rev esc enferm USP [Internet], 45(2), 487-493. doi: 10.1590/S0080-62342011000200026

Schreiter, S., Pijnenborg, G. H. M., \& Rot, M. (2013). Empathy in adults with clinical or subclinical depressive symptoms. Journal of Affective Disorders, 150, 1-16. doi: 10.1016/j.jad.2013.03.009

Wang, M., Wang, J., Deng, X., \& Chen. (2019). Why are empathic children more liked by peers? The mediating roles of prosocial and aggressive behaviors. Personality and Individual Differences, 144, 19-23. doi: 10.1016/j.paid.2019.02.029.

World Health Organization (2017). World Health Day 2017 (Campaign). Retrieved from: http://www.who.int/campaigns/world-health-day/2017/campaign-essentials/en/ 
Financiamento: Este trabalho recebeu apoio da Fundação de Amparo à Ciência e Tecnologia do Estado de Pernambuco - FACEPE e da Coordenação de Aperfeiçoamento de Pessoal de Nível Superior - CAPES.

Participação dos autores: a) Planejamento e concepção do trabalho; b) Coleta de dados; c) Análise e interpretação de dados; d) Redação do manuscrito; e) Revisão crítica do manuscrito.

L. R. S. contribuiu em a, c, d, e; L. C. d. O. em b, c, d; M. F. D. N. P. em d, e.

Editora científica responsável: Dra. Cecilia Cracco 